Article

\title{
Additive Effect on the Structure of PEDOT:PSS Dispersions and Its Correlation with the Structure and Morphology of Thin Films
}

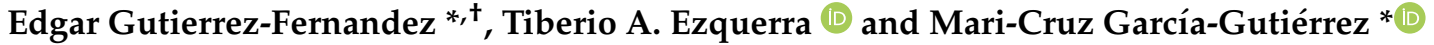 \\ Instituto de Estructura de la Materia (IEM-CSIC), Serrano 121, 28006 Madrid, Spain; t.ezquerra@csic.es \\ * Correspondence: edgar.gutierrez@ehu.eus (E.G.-F.); maricruz@iem.cfmac.csic.es (M.-C.G.-G.) \\ + Present address: POLYMAT, University of the Basque Country UPV/EHU, Av. Tolosa 72, \\ 20018 San Sebastian, Spain.
}

check for updates

Citation: Gutierrez-Fernandez, E.; Ezquerra, T.A.; García-Gutiérrez, M.-C. Additive Effect on the Structure of PEDOT:PSS Dispersions and Its Correlation with the Structure and Morphology of Thin Films. Polymers 2022, 14, 141. https:// doi.org/10.3390/polym14010141 Academic Editor: Jorge Escorihuela Fuentes

Received: 18 November 2021 Accepted: 28 December 2021 Published: 30 December 2021

Publisher's Note: MDPI stays neutral with regard to jurisdictional claims in published maps and institutional affiliations.

Copyright: (C) 2021 by the authors. Licensee MDPI, Basel, Switzerland. This article is an open access article distributed under the terms and conditions of the Creative Commons Attribution (CC BY) license (https:// creativecommons.org/licenses/by/ $4.0 /)$.

\begin{abstract}
We reported on the interaction between poly(3,4-ethylenedioxythiophene):polystyrene sulfonate (PEDOT:PSS) and high-boiling-point additives in PEDOT:PSS aqueous dispersions and in the final polymer films with the aim of stablishing correlations between the structure of both inks and solid thin films. By Small-Angle X-ray Scattering (SAXS) using synchrotron radiation, it was found that the structural changes of dispersions of PEDOT:PSS with high-boiling-point additives can be explained as a two-step mechanism depending on the additive concentration. A compaction of PEDOT:PSS grains was observed at low concentrations while a swelling of the grains together with a phase segregation between PEDOT and PSS segments was evidenced at larger concentrations. Thin films' morphology and structure were investigated by atomic force microscopy (AFM) and synchrotron Grazing Incidence Wide-Angle X-ray Scattering (GIWAXS) respectively. Our two-step model provides an explanation for the small and sharp domains of PEDOT:PSS thin films observed for low-additive concentrations (first step) and larger domains and roughness found for higheradditive concentrations (second step). A reduction of the ratio of PSS in PEDOT:PSS thin films upon the presence of additives was also observed. This can be related to a thinning of the PSS shells of PEDOT:PSS grains in the dispersion. The results discussed in this work provide the basis for a controlled tuning of PEDOT:PSS thin films structure and the subsequent electrical properties.
\end{abstract}

Keywords: PEDOT:PSS; additive; co-solvent; secondary doping; SAXS; thin films

\section{Introduction}

Extensive research has been performed in order to develop electrically conductive polymer materials, either by direct synthesis of conjugated polymers [1] or by the incorporation of conductive nanoadditives into the insulating polymer matrix [2,3]. Among conjugated polymers, poly(3,4-ethylenedioxythiophene):poly(styrenesulfonate) (PEDOT:PSS) is especially interesting because it is dispersed in water, highly conductive, and almost transparent in the visible region when processed as thin film. In addition, it presents high stability at ambient conditions [4]. PEDOT:PSS is formed by the stable, conjugated polymer poly (3,4ethylenedioxythiophene) (PEDOT) which is insoluble in water or in any organic solvent and polystyrene sulfonate (PSS), which is water-soluble and acts as a charge-balancing counter ion. PEDOT:PSS is typically available in aqueous dispersion promoting the formation of micelles. The hydrophobic polycationic PEDOT segments tend to form a core of dispersed grains surrounded by the polyanionic PSS chains, which constitute the hydrophilic shell [4].

Many applications have been accomplished with this polymer. In organic solar cells, PEDOT:PSS is prominent as the hole-extracting layer, enhancing the work function in comparison with the usual transparent electrode indium tin oxide (ITO) and improving the contact between the electrode and the active layer [5]. It is also used in perovskite solar cells [6], OLEDs [7], OFETs [8], and thermoelectrics [9]. Different oxidation levels 
lead to changes in the visible absorption spectra of PEDOT:PSS, making it suitable for electrochromic windows [10]. Besides its high conductivity, PEDOT:PSS is also a pseudocapacitive material, which makes it interesting as a supercapacitor [11]. Controlled stimuli of PEDOT:PSS films to ambient water opens also the possibility of fabricating humidity sensors [12]. Furthermore, its water solubility offers potential applications in biology and medicine [13,14]. However, some of the potential applications for PEDOT:PSS as transparent conducting electrodes require much higher values of electrical conductivity than that provided by the as-received PEDOT:PSS. It is envisioned as a versatile material due to the vast catalogue of physical approaches that has been proved to improve its electrical properties [15]. The incorporation of both liquid [6,16-18] or solid additives [19] into the original water dispersion is indeed a simple and effective approach to enhance the electrical conductivity of the material. Other groups showed that carbon nanofillers improve the thermal stability [20] of PEDOT:PSS, and that the hydrogen-bonding interaction between the matrix and a polar filler could be beneficial as well for this purpose [21]. It is clear that the properties of the final device will depend on the interactions between PEDOT:PSS and the additive: (i) in the dispersion; (ii) during the deposition step; and (iii) in the final postdeposition treatment [22,23]. The full interplay between PEDOT:PSS and liquid additives during the whole procedure is not clearly understood. This work is aimed to clarify the influence of polar additives with a high boiling point into the morphology of dispersed PEDOT:PSS and its subsequent solid-state thin film.

At this moment, the effect of the additive, commonly referred to as "secondary doping", has been explained in terms of its solubility with both the anions of PSS and the neutral segments of polystyrene sulfonic acid (PSSH) [24,25]. This effect leads to conformational changes [26] and crystallinity enhancement of PEDOT oligomers [27,28]. All these effects have been correlated with a phase segregation between segments of PEDOT and PSS, initially strongly bounded by electrostatic forces [29]. Transparent and conducting supported thin films can be prepared from PEDOT:PSS inks [30-32]. Free-standing films can also be prepared by using different deposition procedures including solution cast [33], spin-coating [34,35], electro-spinning [36], or inkjet printing [23,27,37], among others. In all cases, the final properties may strongly depend on film uniformity [38,39], on the thickness [40,41], and roughness [39]. As previously mentioned, several works have focused on the study of properties of deposited thin films of PEDOT:PSS treated with polar solvents $[16,28,42-45]$. The interaction between PEDOT:PSS and polar additives in the aqueous dispersion and in the final thin films has also received some attention. However, only a few reports [46] focused on the correlations between aqueous suspensions and thin films morphology. Thus, it is pertinent to unveil the influence of a modified ink of PEDOT:PSS on the morphology of the deposited thin film in order to develop protocols that will improve the final properties.

In this context, the present work focused on the effect of ethylene glycol (EG), glycerol (G), dimethyl sulfoxide (DMSO), and the blend (G+DMSO) on: (i) the structure of PEDOT:PSS aqueous dispersions and (ii) the structure and morphology of thin films. The structure of the PEDOT:PSS inks was investigated by small-angle X-ray scattering (SAXS) using synchrotron radiation. The results were discussed in terms of different models. Thin films morphology and structure were investigated by atomic force microscopy (AFM) and by synchrotron grazing incidence wide-angle X-ray scattering (GIWAXS) respectively. Finally, correlations between the structure of thin films and inks were established.

\section{Materials and Methods}

PEDOT:PSS aqueous dispersion (Heraeus Clevios ${ }^{\mathrm{TM}}$ AI 4083) was purchased from Ossila Ltd, Sheffield, UK. The concentration of PEDOT:PSS was 1.3\% by weight and the PEDOT:PSS ratio was 1:6. Other chemicals, including ethylene glycol (EG), glycerol (G), and dimethyl sulfoxide (DMSO) were purchased from Sigma-Aldrich (Madrid, Spain) and used as received. The chemical structures of all materials are shown in the Supplementary Information (Figure S1). The PEDOT:PSS aqueous dispersion was filtered through a 
$0.2 \mu \mathrm{m}$ cellulose filter and different additives quantities (in $\mathrm{wt} \%$ ) were added into the PEDOT:PSS dispersion (Table 1) and finally sonicated for $5 \mathrm{~min}$. For the characterization of samples by SAXS in transmission geometry, the dispersions were poured in glass capillaries (Hilgenberg, Malsfeld, Germany, diameter $=1.5 \mathrm{~mm}$, wall thickness $=0.01 \mathrm{~mm}$ ) and then hermetically sealed with hot glue in order to avoid evaporation during the measurements.

Table 1. Additives, additive boiling point, polarity, and sample name, where wt.\% is the weight per cent between the water of the pristine PEDOT:PSS dispersion and the additive.

\begin{tabular}{cccc}
\hline Additive & Boiling Point $\left({ }^{\circ} \mathbf{C}\right)$ & $\begin{array}{c}\text { Polarity in Relation } \\
\text { with Water }\end{array}$ & Sample Name \\
\hline Ethylene Glycol (EG) & 198 & 0.79 & $\mathrm{wt} \% \mathrm{EG}$ \\
\hline Glycerol (G) & 290 & 0.81 & $\mathrm{wt} \% \mathrm{G}$ \\
\hline $\begin{array}{c}\text { Dimethyl Sulfoxide } \\
\text { (DMSO) }\end{array}$ & 189 & 0.44 & $\mathrm{wt} \% \mathrm{DMSO}$ \\
\hline Blend (G+DMSO) & - & - & $\mathrm{wt} \% \mathrm{G}+\mathrm{DMSO}$ \\
\hline
\end{tabular}

Thin films were prepared by spin-coating. Squares of n-silicon wafers (100, Arsenic dopant, Neyco, Vanves, France (France)) were used as substrates, which were sonicated in acetone for $10 \mathrm{~min}$. Subsequently, they were cleaned with isopropyl alcohol for another $10 \mathrm{~min}$ and finally rinsed in deionized water. Then, the substrates were dried with nitrogen blow. A spin-coating equipment (Laurell WS-650 Series by Laurell, North Wales, PA, USA) was used to prepare the thin films. A fixed amount of $0.1 \mathrm{~mL}$ of the dispersion was dropped by a pipette on the substrate and then a rotation rate of $3000 \mathrm{rpm}$ was applied for $60 \mathrm{~s}$. The films were thermally annealed at $\mathrm{T}=140^{\circ} \mathrm{C}$ for $10 \mathrm{~min}$ to remove the additives from the films.

Small-Angle X-ray Scattering (SAXS) experiments were performed at room temperature, at the NCD-SWEET beamline at ALBA synchrotron (Cerdanyola del Vallès, Barcelona, Spain). The X-ray beam with a wavelength $\lambda=0.1 \mathrm{~nm}(\mathrm{E}=12.4 \mathrm{keV})$ impinged the sample in transmission geometry, and the SAXS signal was recorded by a Pilatus $1 \mathrm{M}$ detector located at $2.191 \mathrm{~m}$ from the sample position. Sample-to-detector distance and reciprocal space were calibrated using Silver Behenate. The scattering data were reduced by azimuthal integration of the isotropic 2D SAXS patterns through the whole $q$ range, with $q$ being the modulus of the scattering vector $(q=4 \pi / \lambda(\sin \theta)$, where $2 \theta$ is the scattering angle). The resulting intensity profiles were normalized in terms of photon current and absorbance of the sample, and finally corrected by subtracting the profile from a pure water sample.

Grazing-Incidence Wide-Angle X-ray Scattering experiments (GIWAXS) were performed at ALBA synchrotron, BL-11 (NCD-SWEET). The X-ray beam wavelength was set at $\lambda=0.1 \mathrm{~nm}$. GIWAXS patterns were collected by a LX255-HS 2D (Rayonix) area detector, placed at $11 \mathrm{~cm}$ from the sample. Set-up parameters were calibrated using $\mathrm{Cr}_{2} \mathrm{O}_{3}$.

Atomic Force Microscopy (AFM) measurements were performed with a scanning probe microscope (MultiMode 8 equipped with a Nanoscope V controller, Bruker, Kasruhe, Germany). Topography images were collected in tapping mode using silicon probes (Tap 300GB-G probes by BudgetSensors, Sofia, Bulgaria). The thickness of the films was obtained measuring the height of a scratch. The grain size analysis from the AFM images was performed with ImageJ software using the particles analysis plugin.

Dynamic Light Scattering (DLS) experiments were performed with a Malvern Zetasizer Nano SZ (Malvern Panalytical, Malvern, UK) equipment using disposable folded cuvettes. The PEDOT:PSS dispersion was filtered with a PVDF $200 \mathrm{~nm}$ syringe filter and diluted thirty times.

\section{Results and Discussion}

The diameter of the PEDOT:PSS particles within the aqueous dispersion was first measured by Dynamic Light Scattering experiments (DLS). The results indicated that the dispersion was formed by particles with diameters between $20 \mathrm{nn}$ and $200 \mathrm{~nm}$ whose 
size distribution was centered at $50 \mathrm{~nm}$ (Figure S2). These values are in agreement with other works [47]. It has been suggested that the dispersion stability of PEDOT:PSS is provided by a high concentration of negatively charged PSS monomers in the outer region of the particles, promoting electrostatic repulsion among particles preventing aggregation. Figure 1 presents the intensity profiles as a function of the scattering vector $q$ for the different samples.

(a)

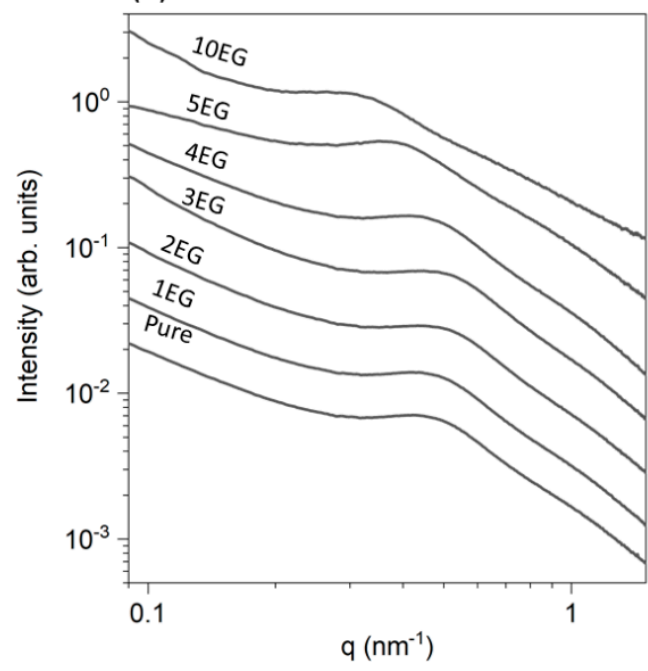

(b)

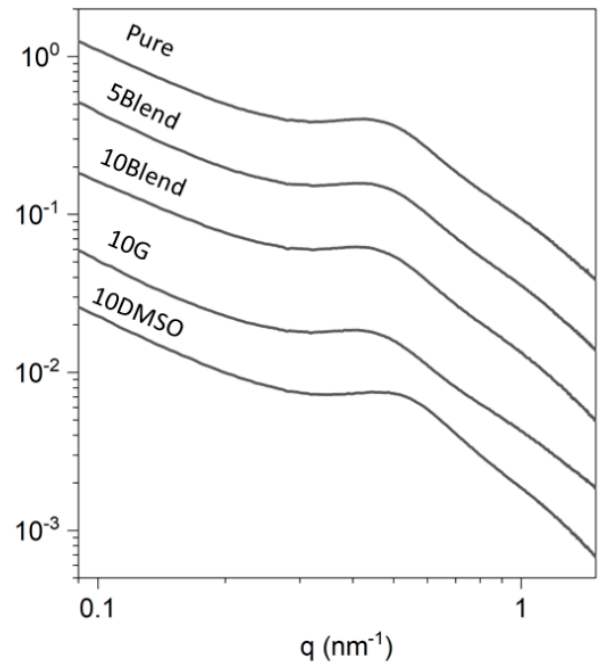

Figure 1. SAXS intensity profiles of PEDOT:PSS (a) with EG and (b) with G, with DMSO and the blend $\mathrm{G}+\mathrm{DMSO}$, at different $\mathrm{w} \mathrm{t} \%$ as labeled. $\mathrm{X}$-axis and $\mathrm{Y}$-axis are presented in logarithmic scale. The profiles are shifted vertically for clarity.

The SAXS profiles from PEDOT:PSS dispersions can be separated in different contributions, depending on the $q$-range [48]: (i) two negative slopes, at low $q\left(\sim 0.1 \mathrm{~nm}^{-1}\right)$ and (ii) another slope at high $q\left(\sim 1 \mathrm{~nm}^{-1}\right)$. This last region is associated with distances around $60 \mathrm{~nm}$ and $6 \mathrm{~nm}$ respectively. In addition, a well-resolved maximum was located at $q \sim 0.4 \mathrm{~nm}^{-1}(d \sim 15 \mathrm{~nm})$. The component at low $q$ originated from the aggregation of colloidal grains, whereas the maximum arose from a correlation length within the grains. The maximum was located at similar $q$ values for all the samples. Thus, we consider that the correlation distance can be associated to a characteristic length within the PEDOT:PSS grains, being that these grains were formed by a PEDOT-rich core and a PSS-rich shell. It has been previously reported that this maximum is related to the well-known polyelectrolyte peak which arises from rod-like segments along the chains as a consequence of the electrostatic repulsion of the highly charged backbone [42,49]. A recent work reported that this maximum is associated with the form factor of the core-shell structure [46]. At a first glance, a shift of the peak maximum to either lower or higher $q$ values with respect to the pristine dispersion, depending on the EG concentration, was observed and is shown in Figure 1a. This effect was less evident in the case of G and DMSO (Figure 1b). The asymptotic behavior of the SAXS intensity at high $q$-values defines what is called the Porod regime. In this regime, the SAXS intensity scattered by a mass fractal follows Porod's law:

$$
I \propto q^{-d_{m}}
$$

where $d_{m}$ is the fractal dimensionality of the scatterer [50,51]. For a one-dimensional randomly oriented rod-like scatterer, $d_{m}=1$, while disk-like objects or a Gaussian random coiled chain display $d_{m}=2$. For three-dimensional objects, Equation (1) is not valid since the scattering from the surface of the object has to be taken into account. In these cases, the 
surface is characterized by a surface fractal dimension, $d_{s}$, which relates the surface area to the size of the scattering object. For these cases, Equation (1) can be generalized as being:

$$
I \propto q^{-\left(2 d_{m}-d_{s}\right)}
$$

For a sharp, non-fractal surface of a three dimensional object, $d_{s}=2$ and $d_{m}=3$; thus, Porod's Law becomes $I \propto q^{-4}$, while less negative exponents were found for rougher, fractal surfaces. The intensity profiles were fitted taking the same $q$-range for all the samples $\left(0.7<q / \mathrm{nm}^{-1}<1.1\right)$. The exponents are listed in Table 2.

Table 2. Decay exponent for every SAXS intensity profile in the high- $q$ range $q=0.7-1.1 \mathrm{~nm}^{-1}$.

\begin{tabular}{cccc}
\hline EG (wt.\%) & Exponent & G (wt.\%) & Exponent \\
\hline 0 & -1.91 & 10 & -1.78 \\
\hline 1 & -1.96 & DMSO (wt \%) & -2.2 \\
\hline 2 & -2.05 & 10 & \\
\hline 3 & -2.10 & G+DMSO (wt $\%)$ & -1.95 \\
\hline 4 & -1.96 & 5 & -1.97 \\
\hline 5 & -1.78 & 10 & \\
\hline 10 & -1.52 & & \\
\hline
\end{tabular}

The intensity profile from a pure PEDOT:PSS dispersion decays as $I \propto q^{-1.91}$, suggesting a random coil chain conformation. Above $4 \mathrm{wt}$ \% $\%$ E concentration, the exponent reduces considerably, suggesting a clear modification of the structure from a random coil to a rod-like scatterer. A similar interpretation can explain the electrostatic interaction between PEDOT:PSS and ionic dopants [46]. On the other hand, while G induced a similar effect as EG at the same concentration, DMSO seemed to induce the opposite, i.e., a more negative exponent.

In order to better visualize the impact of additives on the power decay, we used Kratky plots $\left(I(q) \cdot q^{2}\right.$ vs. $\left.q\right)$ [52] (Figure 2). Kratky plots are commonly used in the analysis of protein solution SAXS to assess the flexibility and shape of the sample. In this case, an absence of slope at high $q$-values can be straightforwardly interpreted as being the characteristics of a random coil Gaussian chain.

(a)

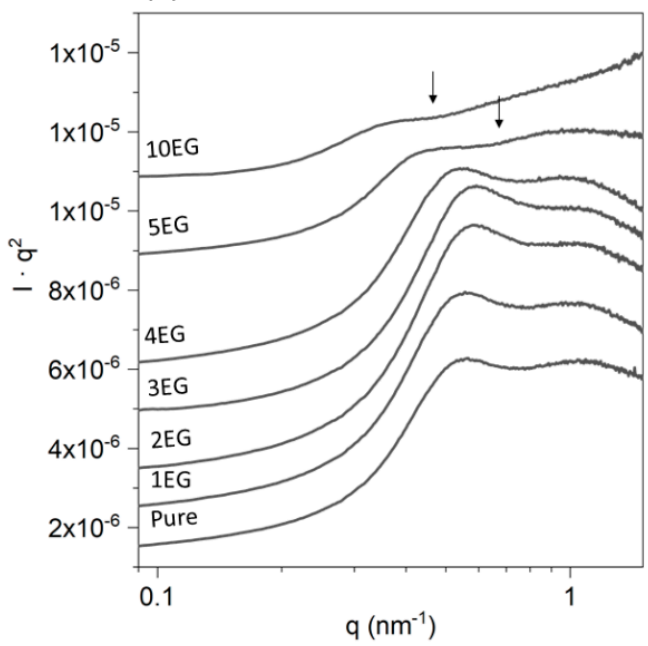

(b)

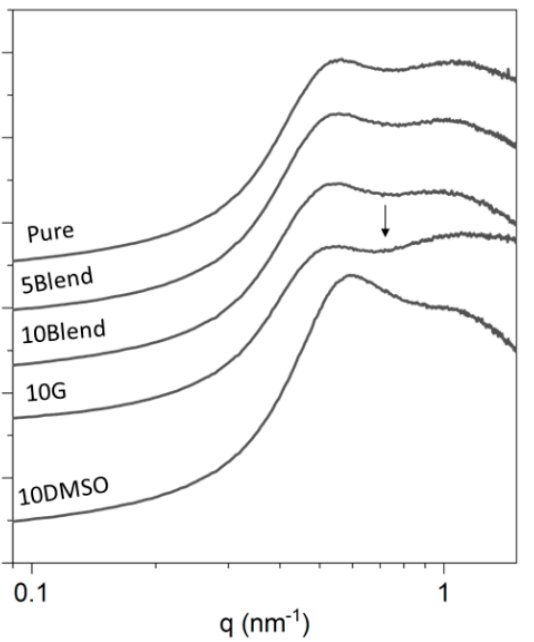

Figure 2. Kratky plots calculated from the SAXS intensity profiles of PEDOT:PSS with EG (a) and with G, with DMSO and G+DMSO (b), at different wt.\% as labelled. The arrows highlight the onset for the upturn of intensity. X-axis are shown in logarithmic scale. The profiles are shifted vertically for clarity. 
The Kratky plot of the pristine dispersion (Figure 2a,b) clearly exhibits the maximum around $q \sim 0.4 \mathrm{~nm}^{-1}$ associated with the polyelectrolyte nature of the sample, followed by a small plateau and a decay for $q>1 \mathrm{~nm}^{-1}$. The typical decay of a random coil structure $\left(I \propto q^{-2}\right)$ should turn into a plateau in the Kratky plot [53].

At $5 \mathrm{wt} . \%$ and higher EG concentrations, the plateau upturns for $q>0.7 \mathrm{~nm}^{-1}$. This effect indicates an intensity decay exponent lower than 2, which can be explained in terms of the persistent chain model [54]. According to this model, for a certain length scale, consecutive chain segments bend with small angles forcing the chain to exhibit a continuous curvature. The relative rigidity of the chain can be quantified by the persistence length. Therefore, if the scattering object is close to a rigid rod, at high $q$-values, the intensity decays by a lower exponent (Equation (1)). In this case, the SAXS profiles should exhibit a transition $q$-value, $q^{*}$ (arrows in Figure 2 ) between the plateau and the upturn. The $q^{*}$ value is related to the persistence length, $a$, by the following expression [54]:

$$
a=\frac{1.91}{q^{*}}
$$

For the PEDOT:PSS dispersion with 5 and $10 \mathrm{wt} \%$ EG (Figure 2a), the $q^{*}$ values can be estimated as being around $0.62 \mathrm{~nm}^{-1}$ and $0.42 \mathrm{~nm}^{-1}$ respectively. These values correspond to persistence lengths of $a \sim 3 \mathrm{~nm}$ and $4.5 \mathrm{~nm}$, respectively. The SAXS profile of the sample with $10 \mathrm{wt} \% \mathrm{G}$ (Figure $2 \mathrm{~b}$ ) also showed an upturn for $q>0.75 \mathrm{~nm}^{-1}(a \sim 2.5 \mathrm{~nm})$. Interestingly enough, DMSO (Figure $2 b$ ) seemed to induce the opposite effect than EG and $\mathrm{G}$, compensating the effect of $\mathrm{G}$ in the samples with the blend G+DMSO.

From the Kratky plot analysis, we can conclude that EG does not induce a significant effect on the structure of aqueous dispersion of PEDOT:PSS until a concentration of $4 \mathrm{wt} \%$ is reached. At this value, PEDOT:PSS chains, which initially arrange according to a random coil configuration, gradually stiffen and behave as rod-like, semi-rigid entities. This effect is reinforced by increasing the EG concentration. The stiffening of the chains is likely to be induced upon the interaction of EG through hydrogen bonding between charged PEDOT and PSS. The polar additive (EG) screens the Coulombic interaction between charged segments which adopt less constrained and more planar conformations. This effect improves the conductivity in PEDOT:PSS thin films [26,29]. While G seems to induce the same effect on the PEDOT:PSS aqueous dispersion, DMSO, by contrast, induces more compact structures. It is noteworthy that EG and $\mathrm{G}$ have a similar relative polarity value (0.79 and 0.81 respectively). These values are higher than that from DMSO (0.44). Thus, it is expected that a concentration of $10 \mathrm{wt} \%$ DMSO has a comparable effect as lower concentrations of EG since DMSO induces a weaker interaction with PEDOT:PSS.

In order to establish quantitative trends upon the incorporation of the additives within the dispersion, the intensity profiles were fitted in the whole $q$-range $\left(0.08-1.5 \mathrm{~nm}^{-1}\right)$ according to an empiric 'broad peak' model [49]. This model has been applied to electrolytic systems [8]. In this case the SAXS intensity is described by:

$$
I(q)=\frac{A_{0}}{q^{n}}+\frac{A_{1}}{1+\left(L \cdot\left|q-q_{\max }\right|\right)^{m}}+A_{2}
$$

where $A_{0}, A_{1}$, and $A_{2}$ are scaling factors, $n$ and $m$ are the low- $q$ and high- $q$ scaling exponents, respectively, and $L$ describes the screening length of the inter-chain interaction [49]. Both $n$ and $m$ exponents describe the dimensionality of the scattering object at different distances. While $n$ is relevant for distances of tens of nanometers, which is of the order of the PEDOT:PSS grain size, $m$ is for distances around 1-10 nm corresponding to the length scale of the inter-chain interactions [49]. The peak, $q_{\max }$, as mentioned before, can be related to a correlation distance $\xi=2 \pi / q_{\text {max }}$ between charged chains due to electrostatic repulsions. All the intensity SAXS profiles can be reasonably fitted to the experimental ones (Figure S3). If PEDOT:PSS grains are visualized as cores of PEDOT with a shell of PSS, then $\xi$ can be interpreted as an average distance between the center of the core (positive region) and 
the middle of the PSS shell (negative region). Figure 3 shows $n$ and $m$ values obtained from the fitting for PEDOT:PSS dispersions with different concentrations of EG, G, DMSO, and G+DMSO.

(a)

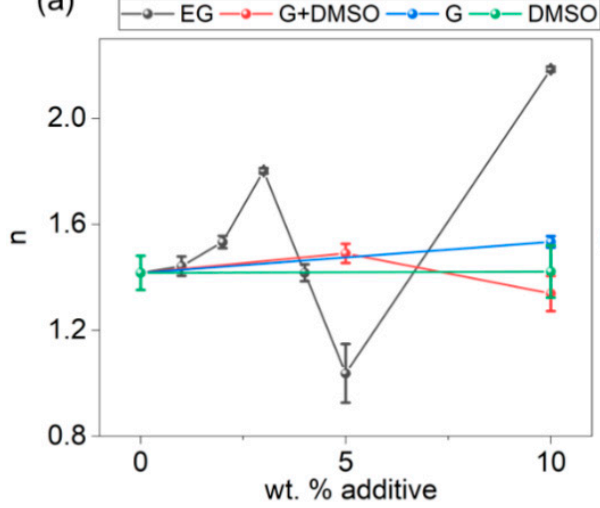

(b)

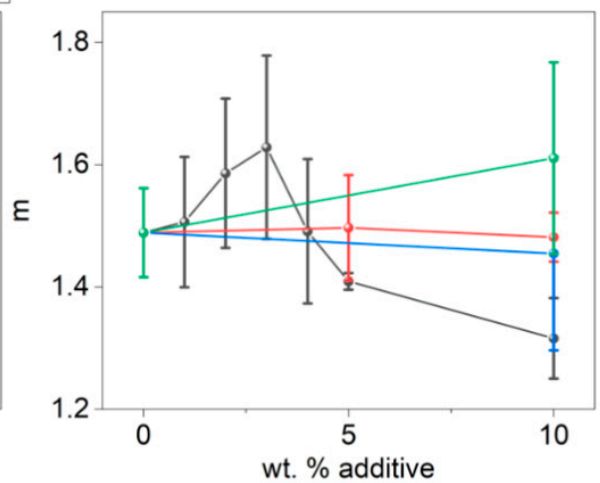

Figure 3. (a) Low- $q$ exponent $n$ and (b) high- $q$ exponent $m$ of PEDOT:PSS dispersions with different concentrations of EG (black points), G (blue points), DMSO (green points), and the blend (G+DMSO) (red points).

The low- $q$ exponents from dispersions of PEDOT:PSS with EG (Figure 3a, black points) tended to increase in most of the concentrations with respect to the pristine value. The $n$ values corresponding to $5 \mathrm{wt} \%$ and $10 \mathrm{wt} \%$ EG were less accurate due to the broadening of the peak in SAXS intensity profiles (Figure 1a) affecting the slope from the aggregates at this $q$-range. The $n$ values for G, DMSO, and the blend were similar in the analyzed range. Concerning the high- $q$ exponent region, a similar trend was observed (Table 2).

The increase of $n$ at low EG concentrations can be associated with the presence of denser aggregates in the dispersions. As above discussed, a reduction of $m$ can be attributed to a transition from a coil-like chain conformation to a more rigid one in the dispersion $[49,55]$. The fitted data from dispersions with EG and G support this behavior. These results are in line with those extracted from Kratky plots concerning the addition of EG to the PEDOT:PSS aqueous dispersion (Figure 2a). A similar conclusion can be suggested for PEDOT:PSS dispersion with G, supporting that the differences can be associated with the high polarity of $\mathrm{G}$. This solvent should interact more efficiently with the charged segments of PSS and PEDOT [55]. Thus, the screening of the Coulombic force would induce a more-extended chain conformation. On the contrary, DMSO seems to affect the PEDOT:PSS dispersion at both the interchain and nanometer distance level as derived from Kratky plots analysis (Figure 2b) but not remarkably affecting the PEDOT:PSS grain structure.

The obtained values for the screening length of the inter-chain interaction, $L \approx 4.5 \pm 0.5 \mathrm{~nm}$, were similar for all systems. Regarding the correlation distance, $\xi=2 \pi / q_{\max }$, the trends for every additive concentration are presented in Figure $4 \mathrm{a}$.

Clearly, EG had a higher interaction with $\xi$ than the other additives. In the case of EG (Figure $4 \mathrm{a}$, black points), $\xi$ dropped slightly from $\xi=14 \mathrm{~nm}$ at low concentrations (for 1-3 wt \%) and increased at higher concentrations, reaching a maximum of $\xi=21 \mathrm{~nm}$. For PEDOT:PSS dispersion with $10 \mathrm{wt} \%$ of G (Figure $4 \mathrm{a}$, blue points), $\xi$ slightly increased compared with that of the pristine value. In the case of $10 \mathrm{wt} \%$ of DMSO (Figure $4 \mathrm{a}$, green points), $\xi$ decreased. The results from dispersions with blend G+DMSO (Figure 4a, red points) presented a slight increase with higher concentrations, showing an intermediate situation between the PEDOT:PSS dispersions with G and DMSO. We can explain the reduction of $\xi$ at low EG concentrations as due to a compaction of the PEDOT:PSS grains, as observed in other polylelectrolyte systems [56]. It is reported that spin-coated thin films of PEDOT:PSS with EG contain less PSS amount than pristine PEDOT:PSS thin films because the EG interacts with the excess of PSS, which is ejected during the spin step [57]. Thus, 
at low concentrations of additive, EG interacts with the PSS shells of PEDOT:PSS grains, removing it partially. The grains would suffer a stronger Coulombic interaction between unbalanced charged regions that would collapse the structure. This would reduce the correlation length between charged zones $\xi$ (Figure 4a, black points) and also could explain the densification of the larger aggregates, as revealed by the increase of the low- $q$ exponent in Figure 3a (black points).

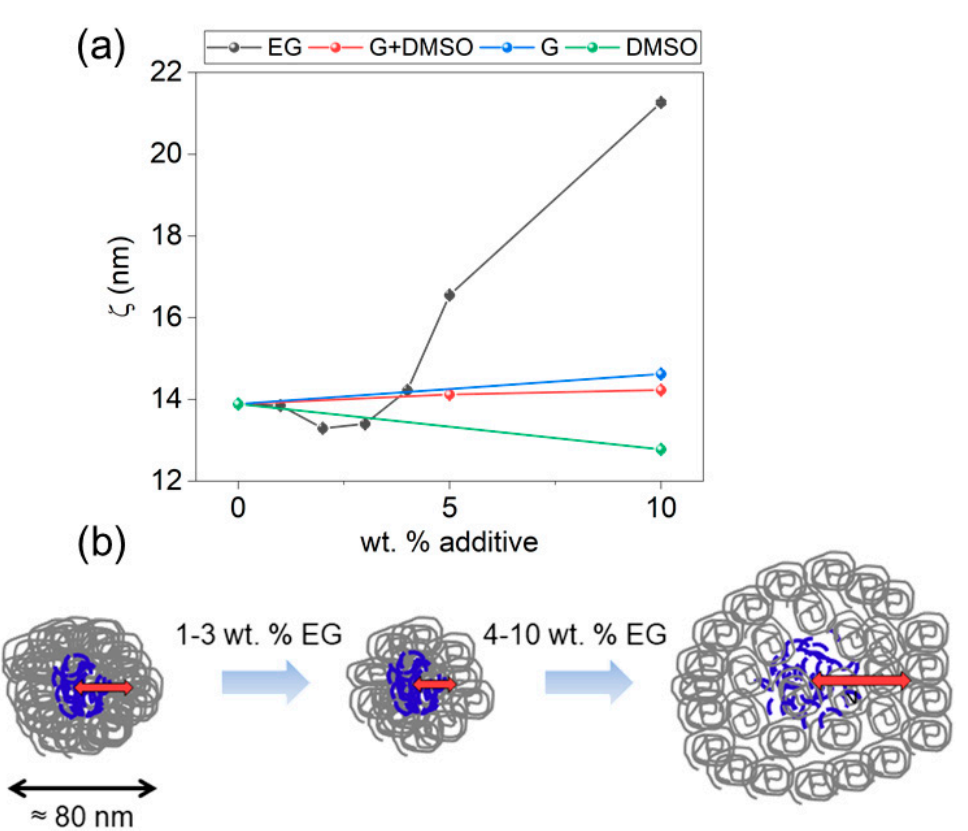

Figure 4. (a) Correlation length $\xi$ for PEDOT:PSS grains at different concentrations of EG (black points), G (blue points), DMSO (green points), and the blend (G+DMSO) (red points). (b) Representation of the effect of EG on the PEDOT:PSS grains within the dispersion. Red arrows represent the idea behind correlation length $\xi$. Scheme not in scale.

The increase of $\xi$ above $4 \mathrm{wt} \%$ EG points toward the swelling of the PEDOT:PSS grains. We explained the two-stage effect of EG considering the scheme of Figure $4 \mathrm{~b}$. At concentrations $\geq 4 \mathrm{wt} \%$, the additive interacts with charged PEDOT and PSS inducing more extended chains and a swelling of the grains in the order of tens of nanometers. This model is coherent with our conclusions based on the SAXS data in the frame of the Kratky plots analysis (Figure 2a, black data). This model is coherent with reported DLS results showing larger PEDOT:PSS grains when the dispersion is mixed with poly(ethylene glycol) [58].

In the case of G, it seemed to follow a similar behavior as EG. However, the effect was less pronounced (Figure $4 \mathrm{a}$, blue data). In the case of DMSO, the trend of $\xi$ exhibited a slight decrease upon the addition of $10 \mathrm{wt} \%$ DMSO. For similar concentrations, Murphy et al. reported almost no change in the SAXS profile, and Bagchi et al. reported an increase of $\xi$ but for much larger concentrations of DMSO.

\section{Additive Effect on the Morphology and Structure of PEDOT:PSS Thin Films}

Thin films from the investigated dispersions were prepared by spin-coating. It was found that DMSO dispersions and EG dispersions with concentrations $>4 \mathrm{wt} \%$ did not form continuous films under the used spin-coating conditions. Figure 5 shows AFM topographical images of pure PEDOT:PSS thin films and mixed with the additives.

The additive induced rougher surfaces from a value $\mathrm{R}_{\mathrm{q}} \sim 1 \mathrm{~nm}$ for a pure PEDOT:PSS thin film to systematically higher values for the different additive-assisted films around $\mathrm{R}_{\mathrm{q}} \sim 2-2.5 \mathrm{~nm}$ (Figure $5 \mathrm{a}-\mathrm{d}$ ). The increase of roughness in PEDOT:PSS films doped with EG can be attributed to either thinning or removal of PSS segments within PEDOT:PSS grains [59-61]. However, looking at the morphology of the AFM images, it is possible to elucidate that the surface consisted of grain-like domains (Figure 5e-h). Independently 
of the additive, an enhancement of the grain sizes was observed. PEDOT:PSS exhibited a distribution of grain sizes centered on $50 \mathrm{~nm}$, in agreement with the DLS experiments (Figure S2). Thin films of PEDOT:PSS with additives exhibited broader and polydisperse distributions ranging from 50 to $200 \mathrm{~nm}$ in diameter (highlighted with a blue arrow in Figure 5i).

(a) $\mathrm{R}_{\mathrm{q}}=1.1 \mathrm{~nm}$

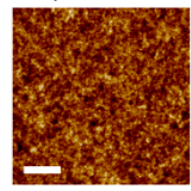

(e)

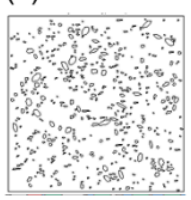

(b) $\mathrm{R}_{\mathrm{q}}=2.3 \mathrm{~nm}$

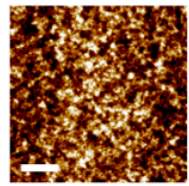

(f)

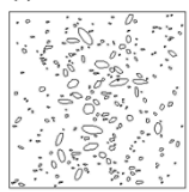

(c) $R_{q}=1.8 \mathrm{~nm}$

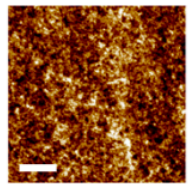

(g)

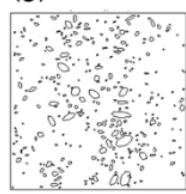

(d) $R_{q}=2.5 \mathrm{~nm}$

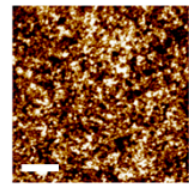

(h)

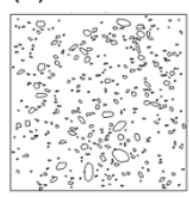

(i)

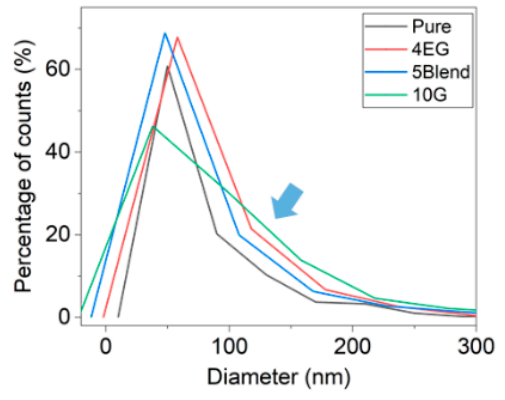

Figure 5. (a-d) AFM topographical images obtained in tapping mode with their corresponding roughness value: (a) pristine PEDOT:PSS, (b) PEDOT:PSS with $4 \mathrm{wt} \%$ EG, (c) PEDOT:PSS with $5 \mathrm{wt} \%$ (G+DMSO) and (d) PEDOT:PSS with $10 \mathrm{wt} \%$ G. (e-h) AFM height images after particle analysis processing. (i) Distribution of grain diameters analyzed by particle analysis plug-in of ImageJ software, version 1.53e.

Smaller domains have been measured by AFM in thicker PEDOT:PSS films with EG [62]. In this case, a reduction of PSS shells during spin-coating is expected. However, enlargement of PEDOT:PSS grains has also been identified by AFM on spin-coated thin films [63]. This effect has been attributed to the coalescence of PEDOT:PSS grains with lower amount of PSS. This apparent contradiction can be understood taking into account that different thermal annealing treatments lead to the coalescence of the grains and the formation of larger domain sizes. The larger grains detected upon mixing with $G$ and G+DMSO may be explained by the swelling of PEDOT:PSS grains after addition of higher additive concentrations, as explained previously. Figure 6 shows the thickness and roughness dependence of PEDOT:PSS thin films as a function of additive concentrations.

(a)

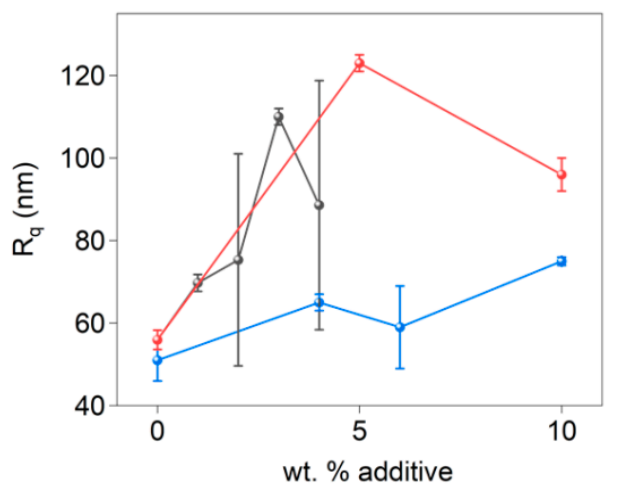

(b)

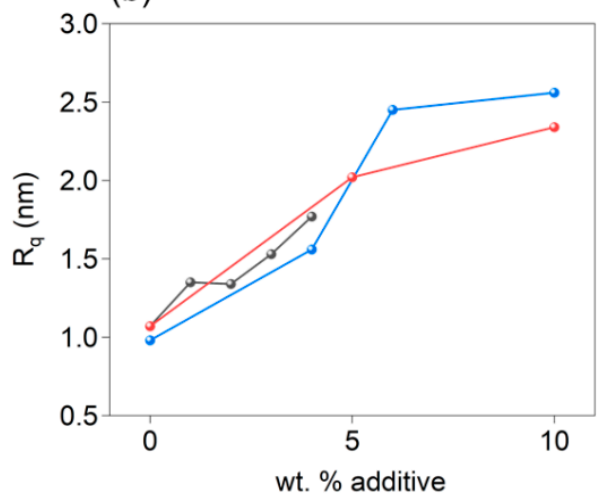

Figure 6. (a) Thickness and $(\mathbf{b})$ roughness $\left(\mathrm{R}_{\mathrm{q}}\right)$ of thin films of PEDOT:PSS with EG (black data), $\mathrm{G}$ (blue data), and G+DMSO (red data).

The thickness of pure PEDOT:PSS thin films is roughly $50 \mathrm{~nm}$. The incorporation of EG increased the thickness, although the film became irregular, as reflected by the size of the error bars. Higher concentrations than $4 \mathrm{wt} \%$ formed discontinuous films. When blended with G or G+DMSO, the thickness also increased as a general trend, although 
it decreased at $10 \mathrm{wt} \%$ of G+DMSO. As previously mentioned, the higher the additive concentration, the rougher the surface (Figure 6b).

The enhanced thickness after deposition and thermal annealing suggest an irreversible reorganization of the material upon the effect of the high-boiling point additive during the deposition. Thermal annealing removes the additive but its effect on the dispersion is retained. In the case of EG, our previous interpretation as a two-stage mechanism can explain why sharper and slightly larger grains were observed in this study and why larger globules are observed by other groups [63]. On the one hand, densification of grains at low EG concentrations provides rougher and granulated surfaces. On the other hand, the swelling detected by SAXS at larger concentrations support larger globules, referred to the structures detected by AFM, around $50-100 \mathrm{~nm}$. In the previous section, larger correlation distances $\xi$ were associated with the swelling of PEDOT:PSS grains. Although continuous thin films of PEDOT:PSS with high EG concentrations were not formed, the swelling induced by $\mathrm{G}$ can be related to the larger domains detected by AFM (Figure 5). This hypothesis can also be applied for thin films of PEDOT:PSS with G+DMSO.

The additive effects on the structure of PEDOT:PSS thin films were elucidated by GIWAXS. Figure 7a-d shows GIWAXS patterns from selected samples. The pattern of pristine PEDOT:PSS (Figure 7a) and with additives (Figure $7 \mathrm{~b}-\mathrm{d}$ ) presented the same reflections at $q=12.5 \mathrm{~nm}^{-1}$ and $q=18.2 \mathrm{~nm}^{-1}$. The first one comes from amorphous PSS, although other works refer to the $\pi-\pi$ stacking of PSS [27]. The one at $q=18.2 \mathrm{~nm}^{-1}$ is associated with the $\pi-\pi$ stacking of PEDOT segments [27].
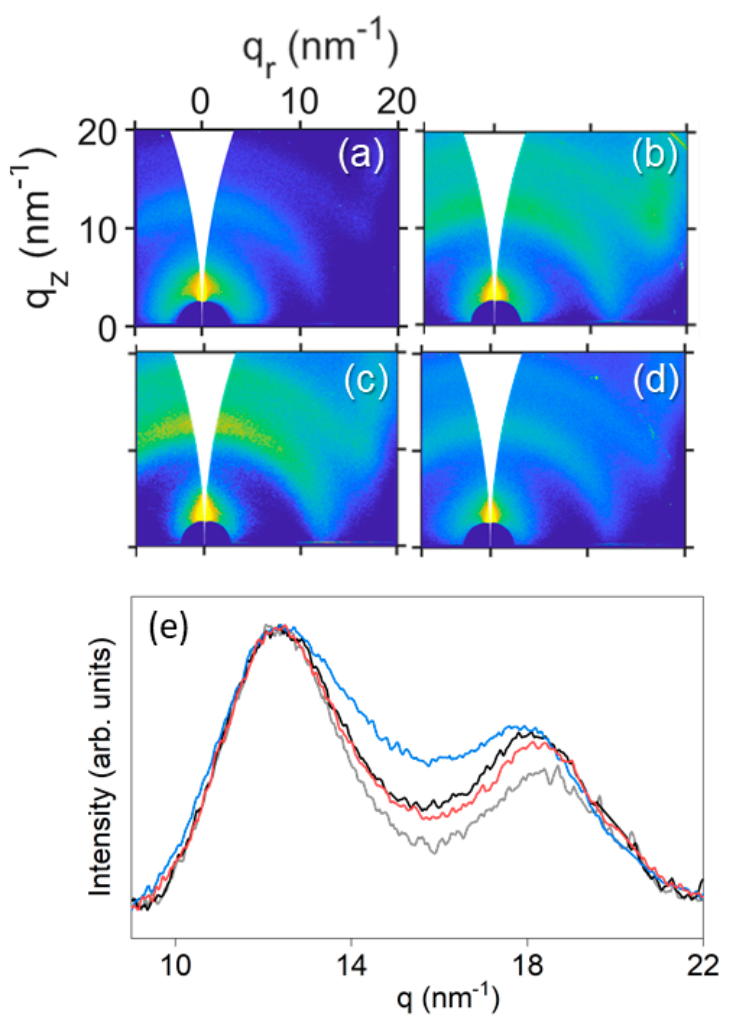

Figure 7. 2D GIWAXS patterns from PEDOT:PSS thin films: (a) pure, (b) 4EG, (c) 10G, and (d) 10G+DMSO. (e) Azimuthal integrations of 2D GIWAXS patterns from pure PEDOT:PSS (gray curve), 4EG (black curve), 10G (blue curve), 10GDMSO (red curve).

No evidence of re-orientation and peak shifting were found, as those reported by Dong et al. [28]. However, we identified relative changes between both Bragg peaks of PSS and PEDOT. We compared the ratios between the peak area associated with PSS and PEDOT. Other groups have seen a PSS reduction in the surface of films upon the interaction of the additive and PSS $[29,57]$. In this work we observed an intensity decrease on the PSS Bragg reflection. In order to compare patterns from different samples in an accurate way, 
the intensity profiles were corrected from background scattering and normalized to the maximum of PSS peaks (Figure 7e). The PEDOT and PSS contributions in the intensity profiles were fitted to Voigt functions and the relation between their areas (APSS / APEDOT) $_{\text {P }}$ are included in Table 3.

Table 3. Ratio of PSS (APSS/APEDOT) in PEDOT:PSS thin films prepared from dispersions with different additives.

\begin{tabular}{lcccc}
\hline & Pristine & $\mathbf{4} \mathbf{w t} \%$ EG & $\mathbf{1 0} \mathbf{w t} \% \mathbf{G}$ & $\mathbf{1 0} \mathbf{w t} \% \mathbf{~ G}+\mathbf{D M S O}$ \\
\hline $\mathrm{A}_{\mathrm{PSS}} / \mathrm{A}_{\text {PEDOT }}$ & $0.98 \pm 0.04$ & $0.79 \pm 0.08$ & $0.80 \pm 0.01$ & $0.91 \pm 0.05$ \\
\hline
\end{tabular}

The ratio of PSS in PEDOT:PSS thin films with the investigated additives was lower than in pristine PEDOT:PSS thin films, being the lowest for EG even with lower concentration. This intensity reduction supports the partial removal of PSS during spin-coating due to interaction of PEDOT:PSS with the additives. As explained for PEDOT:PSS dispersions, the additives induced a phase segregation between PEDOT and PSS, and due to the hydrophilic character of PSS, it was ejected during spin-coating. The reduction of the PSS ratio would improve the conductive properties of PEDOT:PSS thin films [39,48,64].

\section{Conclusions}

High-boiling point additives modify the structure and conformation of PEDOT:PSS water dispersion following a two-step mechanism, depending on additive concentration. A compaction of PEDOT:PSS grains was observed at low concentrations while a higher amount of the additive induced swelling on the grains, possibly due to screening between charged segments of PSS and PEDOT. Thin films formed by the investigated dispersions exhibited a trend of increasing film thickness and roughness with increasing additive concentration. In addition, a direct correlation between the structure of the PEDOT:PSS dispersions and the morphology and internal structure of the corresponding spin-coated thin films was evidenced. The two-stage mechanism described for the effect of additives on the dispersion structure provides an explanation for the small and sharp domains of PEDOT:PSS observed for low additive concentrations (first step), and larger domains and roughness for higher additive concentrations (second step). Finally, the ratio of PSS in PEDOT:PSS thin films with the investigated additives was lower than in pristine PEDOT:PSS thin films, being the lowest for EG even with lower concentrations.

Supplementary Materials: The following supporting information can be downloaded at: https: / / www.mdpi.com/article/10.3390/polym14010141/s1. Chemical structures of the polar additives (Figure S1), distribution of particle diameters by DLS (Figure S2) and fitted SAXS profiles from PEDOT:PSS dispersions (Figure S3).

Author Contributions: Conceptualization, M.-C.G.-G.; methodology, E.G.-F., M.-C.G.-G.; formal analysis, E.G.-F.; writing-original draft preparation, E.G.-F. and M.-C.G.-G.; writing-review and editing, E.G.-F., M.-C.G.-G. and T.A.E.; supervision, M.-C.G.-G. and T.A.E.; project administration, M.-C.G.-G.; funding acquisition, T.A.E. All authors have read and agreed to the published version of the manuscript.

Funding: This research was funded by Spanish State Research Agency (AEI) through projects PID2019-107514GB-I00/AEI/10.13039/501100011033.

Institutional Review Board Statement: Not applicable.

Informed Consent Statement: Not applicable.

Data Availability Statement: The data presented in this study are available on request from the corresponding author.

Acknowledgments: The SAXS and GIWAXS experiments were performed at NCD-SWEET beamline at ALBA synchrotron with the collaboration of ALBA staff: Marc Malfois, Juan Carlos Martínez and Eduardo Solano. 
Conflicts of Interest: The authors declare no conflict of interest.

\section{References}

1. Guo, X.; Facchetti, A. The Journey of Conducting Polymers from Discovery to Application. Nat. Mater. 2020, 19, 922-928. [CrossRef]

2. Zeng, X.-Y.; Zhang, Q.-K.; Yu, R.-M.; Lu, C.-Z. A New Transparent Conductor: Silver Nanowire Film Buried at the Surface of a Transparent Polymer. Adv. Mater. 2010, 22, 4484-4488. [CrossRef]

3. Hernández, J.J.; García-Gutiérrez, M.C.; Nogales, A.; Rueda, D.R.; Kwiatkowska, M.; Szymczyk, A.; Roslaniec, Z.; Concheso, A.; Guinea, I.; Ezquerra, T.A. Influence of Preparation Procedure on the Conductivity and Transparency of SWCNT-Polymer Nanocomposites. Compos. Sci. Technol. 2009, 69, 1867-1872. [CrossRef]

4. Elschner, A.; Kirchmeyer, S.; Lovenich, W.; Merker, U.; Reuter, K. PEDOT. Principles and Applications of an Intrinsically Conductive Polymer; CRC Press: Boca Raton, FL, USA, 2010.

5. Lipomi, D.J.; Tee, B.C.K.; Vosgueritchian, M.; Bao, Z. Stretchable Organic Solar Cells. Adv. Mater. 2011, 23, 1771-1775. [CrossRef]

6. Hu, L.; Li, M.; Yang, K.; Xiong, Z.; Yang, B.; Wang, M.; Tang, X.; Zang, Z.; Liu, X.; Li, B.; et al. PEDOT:PSS Monolayers to Enhance the Hole Extraction and Stability of Perovskite Solar Cells. J. Mater. Chem. A 2018, 6, 16583-16589. [CrossRef]

7. Benor, A.; Takizawa, S.; Pérez-Bolívar, C.; Anzenbacher, P. Efficiency Improvement of Fluorescent OLEDs by Tuning the Working Function of PEDOT:PSS Using UV-Ozone Exposure. Org. Electron. 2010, 11, 938-945. [CrossRef]

8. Horkay, F.; Hammouda, B. Small-Angle Neutron Scattering from Typical Synthetic and Biopolymer Solutions. Colloid Polym. Sci. 2008, 286, 611-620. [CrossRef]

9. Paulraj, I.; Liang, T.F.; Yang, T.S.; Wang, C.H.; Chen, J.L.; Wang, Y.W.; Liu, C.J. Enhanced Power Factor of PEDOT:PSS Films Post-Treated Using a Combination of Ethylene Glycol and Metal Chlorides and Temperature Dependence of Electronic Transport (325-450 K). ACS Appl. Energy Mater. 2020, 3, 12447-12459. [CrossRef]

10. Heuer, H.W.; Wehrmann, R.; Kirchmeyer, S. Electrochromic Window Based on Conducting Poly(3,4-Ethylenedioxythiophene)Poly(Styrene Sulfonate). Adv. Funct. Mater. 2002, 12, 89-94. [CrossRef]

11. Antiohos, D.; Folkes, G.; Sherrell, P.; Ashraf, S.; Wallace, G.G.; Aitchison, P.; Harris, A.T.; Chen, J.; Minett, A.I. Compositional Effects of PEDOT-PSS/Single Walled Carbon Nanotube Films on Supercapacitor Device Performance. J. Mater. Chem. 2011, 21, 15987-15994. [CrossRef]

12. Kang, T.-G.; Park, J.-K.; Yun, G.-H.; Choi, H.H.; Lee, H.-J.; Yook, J.-G. A Real-Time Humidity Sensor Based on a Microwave Oscillator with Conducting Polymer PEDOT:PSS Film. Sens. Actuators B Chem. 2019, 282, 145-151. [CrossRef]

13. Cellot, G.; Lagonegro, P.; Tarabella, G.; Scaini, D.; Fabbri, F.; Iannotta, S.; Prato, M.; Salviati, G.; Ballerini, L. PEDOT:PSS Interfaces Support the Development of Neuronal Synaptic Networks with Reduced Neuroglia Response in Vitro. Front. Neurosci. 2016, 9 , 521. [CrossRef] [PubMed]

14. Gkoupidenis, P.; Schaefer, N.; Garlan, B.; Malliaras, G.G. Neuromorphic Functions in PEDOT:PSS Organic Electrochemical Transistors. Adv. Mater. 2015, 27, 7176-7180. [CrossRef] [PubMed]

15. Shi, H.; Liu, C.; Jiang, Q.; Xu, J. Effective Approaches to Improve the Electrical Conductivity of PEDOT:PSS: A Review. Adv. Electron. Mater. 2015, 1, 1500017. [CrossRef]

16. Palumbiny, C.M.; Heller, C.; Schaffer, C.J.; Körstgens, V.; Santoro, G.; Roth, S.V.; Müller-Buschbaum, P. Molecular Reorientation and Structural Changes in Cosolvent-Treated Highly Conductive PEDOT:PSS Electrodes for Flexible Indium Tin Oxide-Free Organic Electronics. J. Phys. Chem. C 2014, 118, 13598-13606. [CrossRef]

17. Xia, Y.; Sun, K.; Ouyang, J. Solution-Processed Metallic Conducting Polymer Films as Transparent Electrode of Optoelectronic Devices. Adv. Mater. 2012, 24, 2436-2440. [CrossRef]

18. Kim, Y.H.; Sachse, C.; MacHala, M.L.; May, C.; Müller-Meskamp, L.; Leo, K. Highly Conductive PEDOT:PSS Electrode with Optimized Solvent and Thermal Post-Treatment for ITO-Free Organic Solar Cells. Adv. Funct. Mater. 2011, 21, $1076-1081$. [CrossRef]

19. Xia, Y.; Zhang, H.; Ouyang, J. Highly Conductive PEDOT:PSS Films Prepared through a Treatment with Zwitterions and Their Application in Polymer Photovoltaic Cells. J. Mater. Chem. 2010, 20, 9740-9747. [CrossRef]

20. Ahmed, A.; Jalil, M.A.; Hossain, M.M.; Moniruzzaman, M.; Adak, B.; Islam, M.T.; Parvez, M.S.; Mukhopadhyay, S. A PEDOT:PSS and Graphene-Clad Smart Textile-Based Wearable Electronic Joule Heater with High Thermal Stability. J. Mater. Chem. C 2020, 8, 16204-16215. [CrossRef]

21. Wang, X.-y.; Feng, G.-y.; Li, M.-j.; Ge, M.-q. Effect of PEDOT:PSS Content on Structure and Properties of PEDOT:PSS/Poly(Vinyl Alcohol) Composite Fiber. Polym. Bull. 2019, 76, 2097-2111. [CrossRef]

22. Worfolk, B.J.; Andrews, S.C.; Park, S.; Reinspach, J.; Liu, N.; Toney, M.F.; Mannsfeld, S.C.B.; Bao, Z. Ultrahigh Electrical Conductivity in Solution-Sheared Polymeric Transparent Films. Proc. Natl. Acad. Sci. USA 2015, 112, 14138-14143. [CrossRef]

23. Gutiérrez-Fernández, E.; Gabaldón-Saucedo, I.A.; García-Gutiérrez, M.C.; Varea, A.; Nogales, A.; Rebollar, E.; Vilà, A.; Ezquerra, T.A.; Cirera, A. Quantitative Assessment by Local Probe Methods of the Mechanical and Electrical Properties of Inkjet-Printed PEDOT:PSS Thin Films over Indium Tin Oxide Substrates. Org. Electron. Phys. Mater. Appl. 2019, 70, 258-263. [CrossRef]

24. Jönsson, S.K.M.; Birgerson, J.; Crispin, X.; Greczynski, G.; Osikowicz, W.; Denier van der Gon, A.W.; Salaneck, W.R.; Fahlman, M. The Effects of Solvents on the Morphology and Sheet Resistance in Poly(3,4-Ethylenedioxythiophene)-Polystyrenesulfonic Acid (PEDOT-PSS) Films. Synth. Met. 2003, 139, 1-10. [CrossRef] 
25. Xia, Y.; Ouyang, J. PEDOT:PSS Films with Significantly Enhanced Conductivities Induced by Preferential Solvation with Cosolvents and Their Application in Polymer Photovoltaic Cells. J. Mater. Chem. 2011, 21, 4927-4936. [CrossRef]

26. Ouyang, J.; Xu, Q.; Chu, C.W.; Yang, Y.; Li, G.; Shinar, J. On the Mechanism of Conductivity Enhancement in Poly(3,4Ethylenedioxythiophene):Poly(Styrene Sulfonate) Film through Solvent Treatment. Polymer 2004, 45, 8443-8450. [CrossRef]

27. Palumbiny, C.M.; Liu, F.; Russell, T.P.; Hexemer, A.; Wang, C.; Müller-Buschbaum, P. The Crystallization of PEDOT:PSS Polymeric Electrodes Probed in Situ during Printing. Adv. Mater. 2015, 27, 3391-3397. [CrossRef] [PubMed]

28. Dong, J.; Portale, G. Role of the Processing Solvent on the Electrical Conductivity of PEDOT:PSS. Adv. Mater. Interfaces 2020, 7, 2000641. [CrossRef]

29. Mengistie, D.A.; Wang, P.-C.; Chu, C.-W. Effect of Molecular Weight of Additives on the Conductivity of PEDOT: PSS and Efficiency for ITO-Free Organic Solar Cells. J. Mater. Chem. A 2013, 1, 9907-9915. [CrossRef]

30. Vosgueritchian, M.; Lipomi, D.J.; Bao, Z. Highly Conductive and Transparent PEDOT:PSS Films with a Fluorosurfactant for Stretchable and Flexible Transparent Electrodes. Adv. Funct. Mater. 2012, 22, 421-428. [CrossRef]

31. Xia, Y.; Sun, K.; Ouyang, J. Highly Conductive Poly(3\{,\}4-Ethylenedioxythiophene):Poly(Styrene Sulfonate) Films Treated with an Amphiphilic Fluoro Compound as the Transparent Electrode of Polymer Solar Cells. Energy Environ. Sci. 2012, 5, 5325-5332. [CrossRef]

32. Tait, J.G.; Worfolk, B.J.; Maloney, S.A.; Hauger, T.C.; Elias, A.L.; Buriak, J.M.; Harris, K.D. Spray Coated High-Conductivity PEDOT:PSS Transparent Electrodes for Stretchable and Mechanically-Robust Organic Solar Cells. Sol. Energy Mater. Sol. Cells 2013, 110, 98-106. [CrossRef]

33. Lang, U.; Naujoks, N.; Dual, J. Mechanical Characterization of PEDOT:PSS Thin Films. Synth. Met. 2009, 159, 473-479. [CrossRef]

34. Greco, F.; Zucca, A.; Taccola, S.; Menciassi, A.; Fujie, T.; Haniuda, H.; Takeoka, S.; Dario, P.; Mattoli, V. Ultra-Thin Conductive Free-Standing PEDOT/PSS Nanofilms. Soft Matter 2011, 7, 10642. [CrossRef]

35. Lang, U.; Muller, E.; Naujoks, N.; Dual, J. Microscopical Investigations of PEDOT:PSS Thin Films. Adv. Funct. Mater. 2009, 19, 1215-1220. [CrossRef]

36. Zhao, W.; Yalcin, B.; Cakmak, M. Dynamic Assembly of Electrically Conductive PEDOT:PSS Nanofibers in Electrospinning Process Studied by High Speed Video. Synth. Met. 2015, 203, 107-116. [CrossRef]

37. Mannerbro, R.; Ranlöf, M.; Robinson, N.; Forchheimer, R. Inkjet Printed Electrochemical Organic Electronics. Synth. Met. 2008, 158, 556-560. [CrossRef]

38. Natori, A.Y.; Canestraro, C.D.; Roman, L.S.; Ceschin, A.M. Modification of the Sheet Resistance of Ink Jet Printed Polymer Conducting Films by Changing the Plastic Substrate. Mater. Sci. Eng. B Solid-State Mater. Adv. Technol. 2005, 122, $231-235$. [CrossRef]

39. Wilson, P.; Lekakou, C.; Watts, J.F. A Comparative Assessment of Surface Microstructure and Electrical Conductivity Dependence on Co-Solvent Addition in Spin Coated and Inkjet Printed Poly(3,4-Ethylenedioxythiophene):Polystyrene Sulphonate (PEDOT:PSS). Org. Electron. Phys. Mater. Appl. 2012, 13, 409-418. [CrossRef]

40. Eom, S.H.; Senthilarasu, S.; Uthirakumar, P.; Yoon, S.C.; Lim, J.; Lee, C.; Lim, H.S.; Lee, J.; Lee, S.H. Polymer Solar Cells Based on Inkjet-Printed PEDOT:PSS Layer. Org. Electron. Phys. Mater. Appl. 2009, 10, 536-542. [CrossRef]

41. Eom, S.H.; Park, H.; Mujawar, S.H.; Yoon, S.C.; Kim, S.-S.; Na, S.-I.; Kang, S.-J.; Khim, D.; Kim, D.-Y.; Lee, S.-H. High Efficiency Polymer Solar Cells via Sequential Inkjet-Printing of PEDOT:PSS and P3HT:PCBM Inks with Additives. Org. Electron. 2010, 11, 1516-1522. [CrossRef]

42. Ouyang, J. "Secondary Doping" Methods to Significantly Enhance the Conductivity of PEDOT:PSS for Its Application as Transparent Electrode of Optoelectronic Devices. Displays 2013, 34, 423-436. [CrossRef]

43. Ouyang, L.; Musumeci, C.; Jafari, M.J.; Ederth, T.; Inganäs, O. Imaging the Phase Separation between PEDOT and Polyelectrolytes during Processing of Highly Conductive PEDOT:PSS Films. ACS Appl. Mater. Interfaces 2015, 7, 19764-19773. [CrossRef] [PubMed]

44. Cui, H.Q.; Peng, R.X.; Song, W.; Zhang, J.F.; Huang, J.M.; Zhu, L.Q.; Ge, Z.Y. Optimization of Ethylene Glycol Doped PEDOT:PSS Transparent Electrodes for Flexible Organic Solar Cells by Drop-Coating Method. Chin. J. Polym. Sci. Engl. Ed. 2019, 37, 760-766. [CrossRef]

45. Jeong, H.J.; Jang, H.; Kim, T.; Earmme, T.; Kim, F.S. Sigmoidal Dependence of Electrical Conductivity of Thin Pedot:Pss Films on Concentration of Linear Glycols as a Processing Additive. Materials 2021, 14, 1975. [CrossRef]

46. Ju, D.; Kim, D.; Yook, H.; Han, J.W.; Cho, K. Controlling Electrostatic Interaction in PEDOT:PSS to Overcome Thermoelectric Tradeoff Relation. Adv. Funct. Mater. 2019, 29, 1905590. [CrossRef]

47. Sanviti, M.; Alegria, A.; Martínez-Tong, D.E. Fabrication and Nanoscale Properties of PEDOT:PSS Conducting Polymer Nanospheres. ChemRxiv 2021, 1-29.

48. Takano, T.; Masunaga, H.; Fujiwara, A.; Okuzaki, H.; Sasaki, T. PEDOT Nanocrystal in Highly Conductive PEDOT:PSS Polymer Films. Macromolecules 2012, 45, 3859-3865. [CrossRef]

49. Murphy, R.J.; Weigandt, K.M.; Uhrig, D.; Alsayed, A.; Badre, C.; Hough, L.; Muthukumar, M. Scattering Studies on Poly(3,4Ethylenedioxythiophene)-Polystyrenesulfonate in the Presence of Ionic Liquids. Macromolecules 2015, 48, 8989-8997. [CrossRef]

50. Bauer, B.J.; Hobbie, E.K.; Becker, M.L. Small-Angle Neutron Scattering from Labeled Single-Wall Carbon Nanotubes. Macromolecules 2006, 39, 2637-2642. [CrossRef]

51. Schaefer, D.W.; Justice, R.S. How Nano Are Nanocomposites? Macromolecules 2007, 40, 8501-8517. [CrossRef]

52. Roe, R.J. Methods of X-ray and Neutron Scattering in Polymer Science; Oxford University Press: New York, NY, USA, 2000. 
53. Choudhury, P.K.; Bagchi, D.; Sangeeth, C.S.S.; Menon, R. Modified Conformation and Physical Properties in Conducting Polymers Due to Varying Conjugation and Solvent Interactions. J. Mater. Chem. 2011, 21, 1607-1614. [CrossRef]

54. Feigin, L.A.; Svergun, D.I. Structure Analysis by Small-Angle X-ray and Neutron Scattering; Springer: Berlin/Heidelberg, Germany, 1987; Volume 1.

55. Bagchi, D.; Menon, R. Conformational Modification of Conducting Polymer Chains by Solvents: Small-Angle X-ray Scattering Study. Chem. Phys. Lett. 2006, 425, 114-117. [CrossRef]

56. Danielsen, S.P.O.; Sanoja, G.E.; McCuskey, S.R.; Hammouda, B.; Bazan, G.C.; Fredrickson, G.H.; Segalman, R.A. Mixed Conductive Soft Solids by Electrostatically Driven Network Formation of a Conjugated Polyelectrolyte. Chem. Mater. 2018, 30, 1417-1426. [CrossRef]

57. Yan, H.; Okuzaki, H. Effect of Solvent on PEDOT/PSS Nanometer-Scaled Thin Films: XPS and STEM/AFM Studies. Synth. Met. 2009, 159, 2225-2228. [CrossRef]

58. Soleimani-Gorgani, A. Co-Solvents Roles in PEDOT:PSS Ink-Jet Inks. Adv. Nat. Sci. Nanosci. Nanotechnol. 2018, 9, 25009. [CrossRef]

59. Pasha, A.; Khasim, S.; Al-Hartomy, O.A.; Lakshmi, M.; Manjunatha, K.G. Highly Sensitive Ethylene Glycol-Doped PEDOT-PSS Organic Thin Films for LPG Sensing. RSC Adv. 2018, 8, 18074-18083. [CrossRef]

60. Xiao, T.; Cui, W.; Anderegg, J.; Shinar, J.; Shinar, R. Simple Routes for Improving Polythiophene:Fullerene-Based Organic Solar Cells. Org. Electron. Phys. Mater. Appl. 2011, 12, 257-262. [CrossRef]

61. Rahman, Z.A.; Sulaiman, K.; Shuhaimi, A.; Rusop, M. PEDOT:PSS Thin Film as Transparent Electrode in ITO-Free Organic Solar Cell. Adv. Mater. Res. 2012, 501, 252-256. [CrossRef]

62. Unuma, T.; Yoshikawa, M.; Nakamura, A.; Kishida, H. Segmentation of Conducting Domains in PEDOT:PSS Films Induced by an Additive for Conductivity Enhancement. Appl. Phys. Express 2016, 9, 51601. [CrossRef]

63. Singh, V.; Arora, S.; Arora, M.; Sharma, V.; Tandon, R.P. Characterization of Doped PEDOT: PSS and Its Influence on the Performance and Degradation of Organic Solar Cells. Semicond. Sci. Technol. 2014, 29, 45020. [CrossRef]

64. Hu, Z.; Zhang, J.; Zhu, Y. Effects of Solvent-Treated PEDOT: PSS on Organic Photovoltaic Devices. Renew. Energy 2014, 62, 100-105. [CrossRef] 